Botchev, M.A. and Farago, I. and Havasi, A. (2004) Testing weighted splitting schemes on a one-column transport-chemistry model. International journal of environment and pollution, 22 (1/2). pp. 3-16. ISSN 0957-4352

\title{
Testing weighted splitting schemes on a one-column transport-chemistry model
}

\author{
Mike Botchev \\ Dept. of Applied Mathematics, University of Twente, \\ The Netherlands \\ E-mail: m.a.botchev@math.utwente.nl
}

\author{
István Faragó, Ágnes Havasi \\ Dept. of Applied Analysis, Eötvös Loránd University, Hungary \\ E-mails: faragois@cs.elte.hu, hagi@nimbus.elte.hu
}




\title{
Testing weighted splitting schemes on a one-column transport-chemistry model
}

\begin{abstract}
In many transport-chemistry models, a huge system of ODE's of the advection-diffusion-reaction type has to be integrated in time. Typically, this is done with the help of operator splitting. Operator splitting is attractive for complex large-scale transport-chemistry models because it allows to handle different processes separately in different parts of the computer program. Rosenbrock schemes combined with approximate matrix factorization (ROS-AMF) are an alternative to operator splitting which does not suffer from splitting errors. However, since the ROS2-AMF schemes are not based on operator splitting, implementation of these methods often requires serious changes in the code.

In this paper we test another second order splitting introduced by strang in 1963, which seemed to be forgotten and rediscovered recently (partially due to its intrinsic parallellism). This splitting, called symmetrically weighted sequential (SWS) splitting, is simple and straightforward to apply, independent of the order of the operators and has an operator-level parallelism. In the experiments, the SWS scheme compares favorably to the Strang splitting, but is less accurate than ROS-AMF.
\end{abstract}

Keywords: weighted splitting, Strang splitting, ROS-AMF method, transport model, numerical comparison, parallelization.

\section{Introduction}

Transport-chemistry models, describing the concentration changes of different chemical species (so-called tracers) in the atmosphere, are based on a PDE system of the form

$$
\frac{\partial \mathrm{c}_{\mathrm{i}}}{\partial \mathrm{t}}=\mathrm{T}_{\mathrm{i}}\left(\mathrm{c}_{\mathrm{i}}\right)+\mathrm{f}_{\mathrm{i}}\left(\mathrm{c}_{1}, \ldots, \mathrm{c}_{\mathrm{m}}\right), \quad i=1, \ldots, m
$$

appearing, e.g., in Verwer et al. ${ }^{23}$ and Zlatev ${ }^{26}$, where $c_{i}$ denotes the concentration of the $i$ th tracer. The linear differential operator $T_{i}$ describes the various transport processes, such as advection and diffusion, and in global models also cumulus convection. The non-linear term $f_{i}$ represents chemical reactions often including emission and deposition processes.

Since, after spatial discretization, the number of grid-points in a modern air pollution model can range from a few thousand to a few hundred thousands, and the number of chemical species can reach a hundred, the numerical integration of this system on long time intervals is a 
huge computational task. The requirements for accuracy and efficiency can hardly be satisfied if the terms on the right-hand side are treated together. Moreover, these terms have different mathematical properties. For example, the chemistry and - to a smaller extent - the vertical transport operators introduce stiffness to the system and thus require the application of a special implicit method. However, applying an implicit method to the whole problem would be too expensive. This difficulty is usually avoided by using some kind of operator splitting, where the different physical processes are treated separately.

Several splitting methods have been constructed and used in various fields of applied mathematics. In addition to air pollution modelling $\left(\right.$ Zlatev $\left.^{26}\right)$, some areas of application are circulation models (Lanser et al. ${ }^{12}$ ), cloud physics (Marchuk ${ }^{14}$ ) and bio-mathematics (Gerisch and Verwer $^{7}$ ). The simplest kind is sequential splitting, which, in terms of the local splitting error, is first order accurate in time. A second order and therefore more popular method is Strang splitting $\left(\right.$ Marchuk $^{14}$, Strang $\left.^{20}\right)$. Higher order Strang-like splitting schemes were constructed by Yoshida ${ }^{25}$. Since conditions under which the splitting error disappears are not realistic (Dimov et al. ${ }^{6}$, Lanser and Verwer ${ }^{11}$, splitting normally introduces an additional error which may be harmful, especially when one or more split operators is/are stiff. Therefore attempts have been made to come up with a scheme which would not suffer from splitting errors and be as cheap as splitting schemes. One such alternative is the so-called source splitting, in which at each time step, first, one of the operators is advanced in time, and the solution update is applied as a constant source when the other operators are advanced in time (Knoth and Wolke ${ }^{9,10}$ ). In this way splitting is formally removed from the numerical scheme. First proposed by Verwer et al. ${ }^{24}$ combination of Rosenbrock schemes with approximate matrix factorization (AMF) for the Jacobian approximation leads to another class of formally splitting-free methods which are typically more accurate than splitting schemes but do not require any extra costs (Berkvens et al. ${ }^{1}$, Blom and Verwer $^{2}$, Bochev and Verwer ${ }^{3}$, Lanser et al. ${ }^{12}$, Lastdrager et al. ${ }^{13}$ ). Splitting schemes, however, remain to be very popular, probably because of their algorithmic simplicity (a feature crucial in modern, complex models). This also gives possibility of an easier parallelization, for example, using special existing parallel solvers for the sub-problems.

In 1963 Strang proposed a splitting method where a weighted sum of splitting solutions, obtained by different ordering of the sub-operators, are computed at each time step (Strang ${ }^{19}$ ). Analysis of this method can be found in Csomós et al. ${ }^{4}$, Hundsdorfer ${ }^{8}$, Swayne ${ }^{21}$. The symmetrically weighted sequential (SWS) splitting is second order accurate, just like the popular 
Strang splitting. It has also been proven Csomós et al. ${ }^{4}$ that under some circumstances (however mostly unrealistic in real applications) the order can be higher than second. These properties suggest that the weighted splitting schemes may be a good alternative to the traditional splitting methods.

The main aim of this paper is to test the performance of these rediscovered splitting schemes in a simplified one-column version of a global transport-chemistry model. We address the following questions:

- How does the symmetrically weighted splitting compare to the also second order Strang splitting?

- How do these splitting methods compare to the ROS3-AMF scheme (third order Rosenbrock method with approximate matrix factorization), which proved to be a viable alternative to splitting methods in air pollution modelling (Bochev and Verwer ${ }^{3}$, Verwer et al. ${ }^{23}$ )?

The paper has the following structure. Section 2 describes the ROS3-AMF+ method and the splitting schemes to be compared. In Section 3 a brief description of our test model is given and the results of the numerical comparisons are presented. The SWS splitting has nice parallelization properties, which are discussed in Section 4. Finally, conclusions are drawn in Section 5.

\section{Integration methods}

\subsection{ROS3-AMF+}

ROS-AMF schemes are not splitting schemes, since the decomposition of the processes appears only on the linear algebra level (in AMF). The Rosenbrock time integration methods are a generalization of the well-known Runge-Kutta methods (Dekker and Verwer ${ }^{5}$ ). For the semidiscrete autonomous ODE system

$$
\frac{\mathbf{d u}}{\mathrm{dt}}=\mathbf{F}(\mathbf{u})
$$

the third order Rosenbrock method (Lanser et al. ${ }^{12}$, Lastdrager et al. ${ }^{13}$ ) reads as 


$$
\begin{aligned}
& \mathbf{u}^{n+1}=\mathbf{u}^{n}+\frac{5}{4} \mathbf{k}_{1}+\frac{3}{4} \mathbf{k}_{2} \\
& (\mathbf{I}-\gamma \Delta t \mathbf{J}) \mathbf{k}_{\mathbf{1}}=\Delta t \mathbf{F}\left(\mathbf{u}^{n}\right) \\
& (\mathbf{I}-\gamma \Delta t \mathbf{J}) \mathbf{k}_{2}=\Delta t \mathbf{F}\left(\mathbf{u}^{n}+\frac{2}{3} \mathbf{k}_{1}\right)-\frac{4}{3} \mathbf{k}_{1}
\end{aligned}
$$

where $\mathbf{J}$ denotes the Jacobian matrix $\mathbf{F}^{\prime}\left(\mathbf{u}^{n}\right)$ and $\gamma=\frac{1}{2}+\frac{\sqrt{3}}{6}$. We remark that this specific $\gamma$ yields A-stability, which is a highly desirable property if stiff problems are to be solved Lastdrager et al. ${ }^{13}$. In our case the vector $\mathbf{u}$, approximating the concentration function has $m n_{z}$ entries, where $n_{z}$ is the number of vertical layers. Further, $\mathbf{F}(\mathbf{u})=\mathbf{V u}+\mathbf{r}(\mathbf{u})+\mathbf{E}$, where $\mathbf{V}$ is the vertical mixing matrix, $\mathbf{r}$ - the semi-discrete chemical operator, $\mathbf{E}$ is the emission term, and $\mathbf{J}=$ $\mathbf{V}+\mathbf{R}$ with $\mathbf{R}=\frac{\partial \mathbf{r}}{\partial \mathbf{u}}\left(\mathbf{u}^{n}\right)$. There exist modifications of the above scheme in which $\mathbf{I}-\gamma \Delta t \mathbf{J}$ is replaced by an approximate matrix. When standard AMF is used,

$$
(\mathbf{I}-\gamma \Delta t \mathbf{J}) \approx(\mathbf{I}-\gamma \Delta t \mathbf{R})(\mathbf{I}-\gamma \Delta t \mathbf{V})
$$

Such approximations allow to save computational work for the solution of the linear systems with respect to $\mathbf{k}_{\mathbf{1}}$ and $\mathbf{k}_{\mathbf{2}}$ in (3). Note that in large-scale transport-chemistry models solution of the systems with "exact" I $-\gamma \Delta t \mathbf{J}$ would often be computationally unfeasible. The error of the above approximation is $(\gamma \Delta t)^{2} \mathbf{R V}$, which may be large. Therefore, an improved version of this scheme was developed, which is called ROS3-AMF+ Bochev and Verwer ${ }^{3}$. Here the approximation

$$
(\mathbf{I}-\gamma \Delta t \mathbf{J}) \approx\left(\mathbf{L}_{\mathbf{V}}-\gamma \Delta t \mathbf{R}\right) \mathbf{U}_{\mathbf{V}}
$$

is used, with the LU factors of $\mathbf{I}-\gamma \Delta t \mathbf{V}=\mathbf{L}_{\mathbf{v}} \mathbf{U}_{\mathbf{v}}$, $\operatorname{diag} \mathbf{U}_{\mathbf{v}}=\mathbf{I}$. This approximation still has an error of $O\left(\Delta t^{2}\right)$, but it often can be shown to be bounded by $\gamma \Delta t\|\mathbf{R}\|$. Numerical experiments also show that this method is more accurate than standard AMF, while it requires the same computational costs Bochev and Verwer ${ }^{3}$.

\section{2 Sequential and Strang splittings}


Let $\Phi_{V}\left(t_{n}, \Delta t\right)$ and $\Phi_{R}\left(t_{n}, \Delta t\right)$ denote the solution operators applied to the sub-systems

$$
\frac{d y_{1}}{d t}=V y_{1} \text { and } \frac{d y_{2}}{d t}=r\left(y_{2}\right)+E
$$

describing vertical mixing and chemistry with emission, respectively, on the interval $\left(t_{n}, t_{n+1}\right]$. (Ususally, $\Phi_{V}\left(t_{n}, \Delta t\right)$ and $\Phi_{R}\left(t_{n}, \Delta t\right)$ mean numerical solution operators. However, in some cases the sub-problems can be solved exactly and then they can be considered as the continuous solution operators. In the latter case the solution is only affected by the splitting error.) The solution $\mathbf{y}^{n+1}$ of the sequential splitting at $t_{n+1}$ can be expressed a

$$
\mathbf{y}^{n+1}=\Phi_{R}\left(t_{n}, \Delta t\right) \Phi_{V}\left(t_{n}, \Delta t\right) \mathbf{y}^{n}
$$

The ordering of $\Phi_{R}$ and $\Phi_{V}$ in (7) can be changed, however, according to Sportisse and Djouad $^{18}$ in the stiff case this may lead to a decrease of accuracy in the numerical solution.

A splitting scheme with second order local error was suggested by Strang ${ }^{20}$. The Strang splitting, ending e.g., with the vertical mixing operator is defined as

$$
\mathbf{y}^{n+1}=\Phi_{V}\left(t_{n+1 / 2}, \frac{1}{2} \Delta t\right) \Phi_{R}\left(t_{n}, \Delta t\right) \Phi_{V}\left(t_{n}, \frac{1}{2} \Delta t\right) \mathbf{y}^{n}
$$

In the numerical comparisons we modify this scheme as

$$
\mathbf{y}^{n+1}=\Phi_{V}\left(t_{n+1 / 2}, \frac{1}{2} \Delta t\right) \Phi_{R}\left(t_{n+1 / 2}, \frac{1}{2} \Delta t\right) \Phi_{R}\left(t_{n+1 / 2}, \frac{1}{2} \Delta t\right) \Phi_{V}\left(t_{n}, \frac{1}{2} \Delta t\right) \mathbf{y}^{n}
$$

which does not change the second order accuracy of (8). This assures that the method equals the reference method in computational costs per time step. 


\subsection{Weighted sequential splitting}

Another splitting scheme can be obtained by applying sequential splitting in both orders of the sub-operators and by taking a weighted average of the results in each time step according to the following formula:

$$
\mathbf{y}^{n+1}=\Theta\left(\Phi_{V}\left(t_{n}, \Delta t\right) \Phi_{R}\left(t_{n}, \Delta t\right)\right) y^{n}+(1-\Theta)\left(\Phi_{R}\left(t_{n}, \Delta t\right) \Phi_{V}\left(t_{n}, \Delta t\right)\right) \mathbf{y}^{n}
$$

where $\Theta \in(0,1)$ is a weight parameter. This method has second order when $\Theta=0.5$, otherwise first order. For this choice the method is called symmetrically weighted sequential (SWS) splitting, first proposed in Strang ${ }^{19}$. The properties of this scheme on the continuous level were analyzed in Csomós et al. ${ }^{4}$

\section{Numerical comparisons}

\section{1 Model description}

For testing the performance of the methods discussed in Section 2, we chose a simple onecolumn model. The chemical scheme of this model is CBM-IV (Carbon Bond Mechanism IV), involving chemical reactions of 32 species. Emissions are set according to the CBM-IV urban scenario (Sandu et al. ${ }^{16}$ ), which means that the emissions are high. The vertical mixing involves vertical diffusion and convection according to the TM3 global chemistry-transport model $\left(\right.$ Swayne $\left.{ }^{21}\right)$. The number of vertical layers is 19 . Advection is not taken into account.

In our experiments the model is run for a period of five days starting with an initial concentration vector, taken as in http://www.phys.uu.nl ${ }^{22}$. The vertical mixing matrix is updated in every six hours. During the time integration, small negative concentration values occasionally arise, therefore the so-called clipping is used $\left(\mathrm{Sandu}^{17}\right)$. The reference solution in our experiments is obtained by using a very small time-step size. The sub-problems in the splitting schemes were solved by the ROS3 method.

\subsection{Results}


In our comparisons we used time step $\Delta t=15 \mathrm{~min}$ for all the methods. The computational costs were the same for all the methods compared. Since the errors are largest in the surface layer, our observations are mostly based on this layer.

We remark that in the Strang splitting the solution depends considerably on the order of the operators, i.e., in the splitting (9) we could change the order of operators V-R-R-V to R-V-VR. Indications in the literature concerning which order should be taken are ambiguous: Sportisse and Djouad ${ }^{18}$ advocates ending the process with the stiff operator, while Verwer et al. ${ }^{23}$ suggest the other way for the Strang splitting. Therefore, both Strang splittings, Strang V-R-R-V and Strang R-V-V-R were included into the experiments.

We can conclude that generally all the methods, ROS3-AMF+, SWS splitting, Strang VR-R-V and Strang R-V-V-R give good results. The relative errors remain below $10 \%$ in most of the integration time and for most species. The most accurate method is unquestionably ROS3$\mathrm{AMF}+$ for all of the tracers. The fact that the method which is not based on splitting appeared to be the best one, conjectures the crucial role of the splitting error in the global one. Among the other three methods, which all are based on splitting, it is difficult to find a clear winner. The Strang V-R-R-V method could be preferred to the SWS splitting and the other Strang method. The quality of the SWS solutions can be placed between those of the two Strang solutions. A typical case is shown in Figure 1 for layer 1 and in Figure 2 for layer 5.

\section{Place Figure 1 and 2 about here}

More precisely, Strang V-R-R-V was better than Strang R-V-V-R for 20 tracers and than SWS for 18 tracers. SWS was better than Strang R-V-V-R for 21 tracers. It is interesting to examine also the number of those cases where the errors were significant:

- Comparing Strang V-R-R-V versus SWS splitting we see 10 tracers for which one of the schemes gave large errors (from which SWS is more accurate for 7 tracers).

- Comparing Strang R-V-V-R versus SWS splitting we see 11 tracers for which one of the schemes gave large errors (from which SWS is more accurate for 8 tracers). 
We can state that for the most problematic stiff species the SWS splitting performs remarkably well. For three tracers, $\mathrm{OH}, \mathrm{HO}_{2}$ and $\mathrm{NO}_{3}$, the SWS splitting gave much better results than any of the Strang splittings. Figure 3 shows the results obtained for $\mathrm{OH}$.

\section{Place Figure 3 about here}

In the experiments made with Strang R-V-V-R we found two cases where the results were unacceptable: for $\mathrm{N}_{2} \mathrm{O}_{5}$ and $\mathrm{NO}_{3}$, where the correct trend of the concentration changes was not reflected: there was no sign of the high peaks shown by the reference solution. Meanwhile, the SWS splitting was able to describe these peaks, see Figure 4. We can conclude that SWS splitting is not only generally better than Strang R-V-V-R, but, being free from some big errors produced by that method, is also more reliable. This feature should be appreciated all the more because, as we already mentioned, in many cases it is not possible to decide, which Strang method would give better results.

\section{Place Figure 4 about here}

Returning to the question of a proper ordering of the sub-operators in the Strang splitting, we note that in our case the choice proposed in Verwer et al. ${ }^{23}$, namely V-R-R-V, was better than the other one, advocated in Sportisse and Djouad ${ }^{18}$.

\section{Parallelization of the SWS splitting}

If several processors are used, the SWS splitting can be advantageous also from the viewpoint of the CPU time. All the methods considered in this paper can be parallelized across the space, using domain decomposition approach. However, since processes V-R and R-V can be computed independently, the SWS scheme has also a so-called parallelism across the scheme, which, in combination with the parallelism across the domain, leads to an attractive parallel algorithm. This across-the-scheme parallelization has a scalability factor two, i.e., 


$$
\frac{T_{\mathrm{SWS}}}{\hat{T}_{\mathrm{SwS}}}=2,
$$

where $T_{\mathrm{SwS}}(p)$ denotes the CPU time for the SWS splitting parallelized across the space on $p$ processors, and $\hat{T}_{\text {SWS }}(2 p)$ is the CPU time for SWS splitting parallelized across the space and across the method, on $2 p$ processors. The across-the-space parallelization for both Strang splitting and SWS splitting can be characterized by the speedup function

$$
S(B, p)=\frac{p}{B p+1-B},
$$

where $B \in(0,1)$ is the non-parallelizable fraction of the work in the algorithm. The parallel part requires $(1-B) T(1) / p$ time. Thus,

$$
T_{\mathrm{Str}}(2 p)=\frac{T_{\mathrm{Str}}(1)}{S(B, 2 p)} \text {, and } \frac{T_{\mathrm{SWS}}(1)}{T_{\mathrm{SWS}}(p)}=S(B, p),
$$

where $T_{\mathrm{Str}}(2 p)$ is the CPU time of the Strang splitting on $2 p$ processors. By use of (11),

$$
\hat{T}_{\mathrm{SWS}}(2 p)=\frac{T_{\mathrm{SWS}}(1)}{2 S(B, p)} .
$$

We know that if the traditional Strang splitting is used (one middle step according to (8)), then

$$
\frac{T_{\mathrm{SWS}}(1)}{T_{\mathrm{Str}}(1)}=\frac{4}{3} .
$$

(Here we assumed that the two sub-problems are solved equally fast.) It is easy to see that

$$
\hat{T}_{\mathrm{SWS}}(2 p)<T_{\mathrm{Str}}(2 p)
$$

whenever

$$
\frac{4}{3}<\frac{2 S(B, p)}{S(B, 2 p)}=\frac{1-B+2 B p}{1-B+B p},
$$

where the right-hand side increases monotonically to 2 as $p \rightarrow+\infty$. Consequently, if 


$$
p \geq p_{\text {crit }}=\left[\frac{1-B}{2 B}\right]+1,
$$

then for $2 p$ processors SWS splitting is more efficient than Strang splitting. In Figure 5 we plot the predicted CPU times for Strang and SWS splitting versus number of processors for the case $B=0.15$. We see that SWS splitting is faster than Strang already on 6 processors.

Note that ROS3-AMF+ scheme has the same parallelism as Strang splitting.

\section{Place Figure 5 about here}

\section{Conclusions}

We compared the solutions of ROS3-AMF+, SWS splitting and Strang V-R-R-V and R-V-V-R splittings in a one-column transport model with vertical mixing and stiff chemistry. Our main conclusions are as follows.

- All the methods (which are equal in computational costs) give good results with relative errors mostly below $10 \%$ This is often acceptable in modern transport-chemistry models.

- ROS3-AMF+ gives the best results. Strang V-R-R-V splitting performs generally better than SWS splitting, while Strang R-V-V-R splitting is least accurate with unacceptably big errors for two tracers.

- SWS splitting gives acceptable solutions for all species. Also, for most of the problematic stiff species it performs better than any of the Strang splittings. Therefore, since it is generally not known which Strang splitting should be used, the SWS splitting can be a fairly reliable alternative.

- As opposed to Strang splitting, SWS splitting can be parallelized on the operator level, which, in combination with across-the-space parallelism, leads to an attractive parallel algorithm.

- SWS splitting is easy to implement. Switching from Strang splitting to SWS requires only minor modifications in the computer program. 
Acknowledgements. We thank Jan Verwer for pointing us several references. This work was done in the framework of a joint project, sponsored by the Netherlands Organization for Scientific Research (NWO) and the Hungarian Scientific Research Fund (OTKA).

\section{References}

[1] Berkvens, P. J. F., Botchev, M. A., Krol, M. C., Peters, W., Verwer, J. G. (2002) Solving vertical transport and chemistry in air pollution models, IMA volumes in Mathematics and its Applications 130, Atmospheric Modeling, Eds: D. P. Chock and G. R. Carmichael, Springer, pp. $1-20$.

[2] Blom, J. G., Verwer, J. G. (2000) A comparison of integration methods for atmospheric transport-chemistry problems, J. Comput. Appl. Math. 126, No. 1-2, pp. 381-396.

[3] Botchev, M. A., Verwer, J. G. (2003) A new approximate matrix factorization for implicit time integration in air pollution modeling, to appear in J. Comp. Appl. Mathematics.

[4] Csomós, P., Faragó, I., Havasi, Á. (2003) Weighted sequential splittings and their analysis, to appear in Comp. Math. Appl.

[5] Dekker, K., Verwer, J. G. (1984) Stability of Runge-Kutta methods for stiff non-linear differential equations, North-Holland Elsevier Science Publishers.

[6] Dimov, I.., Faragó, I., Havasi, Á., Zlatev, Z. (2002) L-commutativity of the operators in splitting methods for air pollution models, Annal. Univ. Sci. Sec. Math. 44, pp. 127-148.

[7] Gerisch, A., Verwer, J. G. (2002) Operator splitting and approximate factorization for taxisdiffusion-reaction models, Appl. Numer. Math. 42, pp. 159-176.

[8] Hundsdorfer, W. (1992) Unconditional convergence of some Crank-Nicolson LOD methods for initial-boundary value problems, Math. Comput. 58, No. 197, pp. 35-53. 
[9] Knoth, O., Wolke, R. (1994) A comparison of fast chemical kinetic solvers in a simple vertical diffusion model. In S.-V. Gryning and M. M. Millán, editors, Vol. X, Plenum Press, pp. 287-294.

[10] Knoth, O., Wolke, R. (1998) Implicit-explicit Runge-Kutta methods for computing atmospheric reactive flows, Appl. Numer. Math. 28, pp. 327-341.

[11] Lanser, D., Verwer, J. G. (1999) Analysis of operator splitting for advection-diffusionreaction problems in air pollution modelling, J. Comput. Appl. Math. 111, No. 1-2, pp. 201-216.

[12] Lanser, D., Blom, J. G., Verwer, J. G. (2001) Time integration of the shallow water equations in spherical geometry, J. Comput. Phys. 171, pp. 373-393.

[13] Lastdrager, B., Koren B., Verwer, J. G. (2001) Solution of time-dependent advectiondiffusion problems with the sparse-grid combination technique and a Rosenbrock solver, Comput. Methods Appl. Math. 1, pp. 86-98.

[14] Marchuk, G.I. (1968) Some application of splitting-up methods to the solution of mathematical physics problems, Aplik. Mat. 13, No. 2, pp. 103-132.

[15] F. Müller, Splitting error estimation for micro-physical-multiphase chemical systems in meso-scale air quality models, Atm. Env. 35, pp. 5749-5764.

[16] Sandu, A., Verwer, J. G. Blom, J. G., Spee, E. J. Carmichael, G. R. (1997) Benchmarking stiff ODE solvers for atmospheric chemistry problems II: Rosenbrock solvers, Atm. Env. 31, pp. 3459-3472.

[17] Sandu, A. (2001) Positive numerical integration methods for chemical kinetic systems, $J$. Comput. Phys. 170, pp. 589-602.

[18] Sportisse, B., Djouad, R. (2002) Some aspects of multi-timescales issues for the numerical 
modeling of atmospheric chemistry, IMA volumes in Mathematics and its Applications 130, Atmospheric Modeling, Eds: D. P. Chock and G. R. Carmichael, Springer, pp. 1-20.

[19] Strang, G. (1963) Accurate partial difference methods I: Linear Cauchy problems, Archive for Rational Mechanics and Analysis 12, pp. 392-402.

[20] Strang, G. (1968) On the construction and comparison of difference schemes, SIAM J. Numer. Anal. 5, No. 3, pp. 506-517.

[21] Swayne, D. A. (1987) Time dependent boundary and iterior forcing in locally onedimensional schemes, SIAM J. Sci. Statist. Comput. 8, pp. 755-767.

[22] http://www.phys.uu.nl/\$^\sim\$peters/TM3/TM3S.html, the TM3 model home page. IMAU, Utrecht University, Utrecht.

[23] Verwer, J. G., Hundsdorfer, W. H., Blom, J. G. (2002) Numerical time integration for air pollution models, Surveys on Mathematics for Industry 10, pp. 107-174.

[24] Verwer, J. G., Spee, E. J., Blom, J. G., Hundsdorfer, W. (1999) A second-order Rosenbrock method applied to photochemical dispersion problems, SIAM J. Sci. Comput. 20, No. 4, pp. 14561480 .

[25] Yoshida, H. (1990) Construction of higher order symplectic integrators, Phys. Lett. A 150, pp. 262-268.

[26] Zlatev, Z. (1995) Computer treatment of large air pollution models. Kluwer Academic Publisher. 


\section{Captions}

Figure 1: Solutions of ROS3-AMF+, Strang V-R-R-V, Strang R-V-V-R and SWS splitting for trace gas isoprene on layer 1.

Figure 2: Solutions of ROS3-AMF+, Strang V-R-R-V, Strang R-V-V-R and SWS splitting for trace gas isoprene on layer 5 .

Figue 3: Solutions of ROS3-AMF+, Strang V-R-R-V, Strang R-V-V-R and SWS splitting for trace gas $\mathrm{OH}$ on layer 1.

Figure 4: Solutions of SWS splitting and Strang R-V-V-R for trace gas $\mathrm{NO}_{3}$ on layer 1.

Figure 5: Comparison of CPU times for SWS splitting and Strang splitting as a function of the number of processors if $T_{\mathrm{Str}}(1)=1$. 

